
abril-junho 2004 .

\title{
Adubação orgânica e altura de corte da erva-cidreira brasileira ${ }^{1}$
}

\author{
Maurício R.A. Santos ${ }^{2}$; Renato Innecco ${ }^{3}$ \\ ${ }^{2}$ Embrapa Agroindústria Tropical, C. Postal 3761, 60511-110 Fortaleza-CE; E-mail: mrasantos@zipmail.com.br; ${ }^{3}{ }^{U F C}$, Dep ${ }^{\text {to }}$ Fitotecnia, \\ C. Postal 12.168, 60356-001 Fortaleza-CE; E-mail: innecco@ufc.br
}

\begin{abstract}
RESUMO
Avaliou-se os efeitos da adubação orgânica e de alturas de corte em plantas de Lippia alba, quimiotipo limoneno-carvona, na produção de matéria seca foliar e de óleo essencial, no Nordeste brasileiro. As doses $0 ; 2$ e $4 \mathrm{~kg} \mathrm{~m}^{-2}$ de adubo foram aplicadas em parcelas subdivididas em cortes de $15 ; 30$ e $45 \mathrm{~cm}$ de altura, agrupadas em blocos casualizados. Foram realizadas colheitas aos 60 e 120 dias após o plantio. A adubação não influenciou significativamente as produções de matéria seca foliar e de óleo essencial. O corte a $45 \mathrm{~cm}$ de altura resultou na maior produção de matéria seca foliar. As maiores concentrações de óleo essencial foram obtidas nos cortes a 30 e $45 \mathrm{~cm}$ de altura.
\end{abstract}

Palavras-chave: Lippia alba, Verbenaceae, óleo essencial.

\begin{abstract}
Organic fertilization and cutting height of Lippia alba

The effects of organic fertilization and cutting height on dry matter and essential oil production from leaves of Lippia alba, limonene-carvone chemotype, were evaluated in the Northeastern Brazilian Region. A split plot experiment was conducted with the organic fertilization $\left(0 ; 2\right.$ and $\left.4 \mathrm{~kg} \mathrm{~m}^{-2}\right)$ as main plots and $15 ; 30$ and $45 \mathrm{~cm}$ cutting height as subplots. First harvest was done 60 days and the second 120 days after planting date. Dry matter and essential oil production were not influenced by fertilization. Highest dry matter production was observed on $45 \mathrm{~cm}$ cutting height. Highest essential oil production was obtained on 30 and $45 \mathrm{~cm}$ cutting height.
\end{abstract}

Keywords: Lippia alba, Verbenaceae, essential oil.

(Recebido para publicação em 24 de março de 2003 e aceito em 24 de janeiro de 2004)

$L$ ippia alba (Mill.) N.E. Brown é uma das espécies medicinais mais utilizadas pela população brasileira, de acordo com a lista publicada pela Central de Medicamentos (CEME), (Angelucci et al., 1990; Ming, 1992). Popularmente conhecida como erva-cidreira, foi incluída em projetos como "Farmácias Vivas", da Universidade Federal do Ceará (Mattos, 2000a) e "Fitoterapia nos Serviços de Saúde", implementado pela Secretaria Estadual de Saúde do Paraná (Ming, 1990), além de alguns projetos desenvolvidos pela Prefeitura de Campinas (SP), que visam oferecer, sem fins lucrativos, assistência farmacêutica fitoterápica às comunidades carentes (Castro, 2001). Porém, a Organização Mundial de Saúde considera fundamental que se realizem investigações experimentais acerca das plantas utilizadas para fins medicinais e de seus princípios ativos, para garantir sua eficácia e segurança terapêutica (Carlos et al., 2000). Paralelamente, se faz necessário o levantamento etnobotânico das espécies medicinais de cada região fitogeográfica do Brasil (Matos, 1997).
É de grande importância que se estabeleçam linhas de ação voltadas para o desenvolvimento de técnicas de manejo ou cultivo (pesquisas fitotécnicas) das plantas com potencial terapêutico, considerando-se a sua utilização pelo homem e a manutenção do equilíbrio dos ecossistemas (Mattos, 2000b). É fundamental que estas técnicas sejam desenvolvidas respeitando-se as condições edafoclimáticas regionais, uma vez que a produção de princípios ativos pelas plantas pode ser intensamente afetada pelo ambiente de cultivo (Retamar, 1977; Zoghbi et al., 1998).

O objetivo deste trabalho foi estabelecer parte da tecnologia de produção de L. alba, quimiotipo limonenocarvona (Matos, 1996), nas condições do nordeste brasileiro, avaliando os efeitos de níveis de adubação orgânica e de altura de corte das plantas na produção de matéria seca foliar, no teor e na composição química do óleo essencial.

\section{MATERIAL E MÉTODOS}

Os estudos de campo foram realizados em área da UFC, no município de
Pentecoste (CE). A região apresenta médias anuais de $26,8^{\circ} \mathrm{C}$ de temperatura, $73 \%$ de umidade relativa do ar e 723,3 $\mathrm{mm}$ de precipitação pluviométrica, o que caracteriza um clima do tipo quente e úmido (Mattos, 2000a). Utilizou-se mudas de erva-cidreira brasileira (Lippia alba Mill. N.E. Brown - Verbenaceae; det.: F.R.S. Pires; exsicata n 21.806 - Herbário Prisco Bezerra/UFC; coleta: Horto de Plantas Medicinais/UFC, 21.02.1995), produzidas por estaquia e mantidas sob sombrite, com nebulização, por um período de 60 dias até serem transplantadas para canteiros de alvenaria com $10,0 \mathrm{~m}^{2}$, no espaçamento de $0,50 \times 0,50 \mathrm{~m}$, com irrigação por aspersão, duas vezes ao dia, por períodos de três horas. O delineamento estatístico foi em parcelas subdivididas, sendo as doses de adubo orgânico: zero, 2 e 4 kg/m² (tratamentos primários, em blocos ao acaso, com 3 repetições); e as alturas de corte: $15 ; 30$ e $45 \mathrm{~cm}$ (tratamentos secundários), em parcelas de $2,0 \mathrm{~m}^{2}$, com 8 plantas cada.

Os substratos foram preparados misturando-se adubo orgânico (Vitasolo) com arenito. Foram retiradas amostras das parcelas, cuja análise resultou, para

1 Extraído da tese de doutorado do primeiro autor, realizada com apoio da CAPES. 
Tabela 1. Efeito da adubação orgânica em plantas de Lippia alba, quimiotipo limoneno-carvona, em duas colheitas (agosto e outubro/2002). Pentecoste (CE), UFC, 2002.

\begin{tabular}{|c|c|c|c|c|c|}
\hline \multirow[b]{2}{*}{ Colheita (dias) } & \multirow{2}{*}{$\begin{array}{c}\text { Dose de Adubo } \\
\left(\mathrm{kg} \mathrm{m}^{-2}\right)\end{array}$} & \multicolumn{4}{|c|}{ Produção } \\
\hline & & $\begin{array}{c}\text { Matéria seca } \\
\left(\mathrm{t} \mathrm{ha} \mathrm{a}^{-1}\right)\end{array}$ & $\begin{array}{c}\text { Óleo } \\
\text { (L ha-1) }\end{array}$ & $\begin{array}{c}\text { Limoneno } \\
\left(\mathrm{L} \mathrm{ha}^{-1}\right)\end{array}$ & $\begin{array}{c}\text { Carvona } \\
\text { (L ha-1) }\end{array}$ \\
\hline 60 & 15 & $0,948 \mathrm{a}$ & $4,715 \mathrm{a}$ & $0,709 a$ & $2,037 \mathrm{a}$ \\
\hline 60 & 30 & $0,871 \mathrm{a}$ & 4,347 a & $0,638 a$ & $1,279 a$ \\
\hline 60 & 45 & $0,692 a$ & $3,477 \mathrm{a}$ & $0,701 \mathrm{a}$ & $1,512 \mathrm{a}$ \\
\hline 120 & 15 & $1,504 \mathrm{a}$ & $11,799 a$ & $1,810 \mathrm{a}$ & 5,015 a \\
\hline 120 & 30 & $1,341 \mathrm{a}$ & $10,851 \mathrm{a}$ & $1,753 \mathrm{a}$ & $2,899 \quad b$ \\
\hline 120 & 45 & $1,306 a$ & $10,090 \mathrm{a}$ & $2,096 \mathrm{a}$ & 3,202 \\
\hline Total & 15 & $2,450 \mathrm{a}$ & $16,514 \mathrm{a}$ & $2,508 \mathrm{a}$ & 7,053 a \\
\hline Total & 30 & $2,212 a$ & $15,198 \mathrm{a}$ & $2,387 a$ & $4,181 \quad b$ \\
\hline Total & 45 & $1,994 a$ & $13,568 a$ & $2,796 a$ & $4,712 \quad b$ \\
\hline
\end{tabular}

*/ Letras diferentes indicam diferença significativa, dentro de cada colheita, com $5 \%$ de probabilidade pelo teste de Tukey.

$0,0 \mathrm{~kg} \mathrm{~m}^{-2}$ de adubo: $\mathrm{pH}=4,3 ; \mathrm{Ca}=1,0$ cmolc dm ${ }^{-3} ; \mathrm{Mg}=1,0$ cmolc $\mathrm{dm}^{-3} ; \mathrm{K}=$ $60,0 \mathrm{mg} \mathrm{dm}^{-3} ; \mathrm{Na}=52,0 \mathrm{mg} \mathrm{dm}^{-3} ; \mathrm{Al}=$ $0,15 \mathrm{cmolc} \mathrm{dm}^{-3}$ e $\mathrm{P}=3,0 \mathrm{mg} \mathrm{dm}^{-3}$; para $2,0 \mathrm{~kg} \mathrm{~m}^{-2}: \mathrm{pH}=4,8 ; \mathrm{Ca}=1,4 \mathrm{cmolc} \mathrm{dm}^{-3}$; $\mathrm{Mg}=1,3 \mathrm{cmolc} \mathrm{dm}^{-3} ; \mathrm{K}=93,0 \mathrm{mg} \mathrm{dm}^{-3}$; $\mathrm{Na}=46,0 \mathrm{mg} \mathrm{dm}^{-3} ; \mathrm{Al}=0,10 \mathrm{cmolc} \mathrm{dm}^{-3}$ e $\mathrm{P}=115,0 \mathrm{mg} \mathrm{dm}^{-3}$; e para $4 \mathrm{~kg} \mathrm{~m}^{-2}: \mathrm{pH}$ $=4,8 ; \mathrm{Ca}=1,4 \mathrm{cmolc} \mathrm{dm}^{-3} ; \mathrm{Mg}=1,4$ cmolc $\mathrm{dm}^{-3} ; \mathrm{K}=121,0 \mathrm{mg} \mathrm{dm}^{-3} ; \mathrm{Na}=$ $64,0 \mathrm{mg} \mathrm{dm}^{-3} ; \mathrm{Al}=0,10 \mathrm{cmolc} \mathrm{dm}^{-3} \mathrm{e} \mathrm{P}=$ $155,0 \mathrm{mg} \mathrm{dm}^{-3}$. Foram realizadas duas colheitas, aos 60 e 120 dias após o transplante das mudas (nos meses de agosto e outubro/2002, respectivamente), em torno das 9 horas da manhã, nas quais se coletou quatro repetições de $500 \mathrm{~g}$ de folhas frescas, as quais foram imediatamente submetidas à extração de óleo essencial por arraste a vapor, utilizando metodologia descrita por Craveiro et al. (1981). As folhas foram secas em estufa, a $45^{\circ} \mathrm{C}$, até atingirem peso constante. Utilizando uma balança analítica, determinou-se a matéria seca das folhas. Os rendimentos de óleo essencial e dos seus constituintes foram obtidos através de CG/EM, de acordo com metodologia descrita por Alencar et al. (1984). Os dados obtidos foram submetidos à análise de variância e as médias foram comparadas pelo teste de Tukey, ao nível de 5\% de significância (Gomes, 1986).

\section{RESULTADOS E DISCUSSÃO}

Não ocorreram diferenças significativas entre os tratamentos de adubação, nas produções de matéria seca foliar e de óleo essencial (Tabela 1). Em relação às duas variáveis estudadas, as produções obtidas na segunda colheita foram superiores às da primeira, provavelmente porque as plantas já estavam plenamente estabelecidas. A produção de limoneno não sofreu efeito da adubação, em nenhuma das colheitas. A produção de carvona foi influenciada negativamente pela adubação: na primeira colheita, os resultados não diferiram significativamente, mas na segunda a ausência de adubação resultou na maior produção de carvona (5,015 L/ha). Este resultado se repetiu no total obtido deste composto nas duas colheitas, perfazendo 7,053 L/ha.

Estes resultados divergem de alguns trabalhos dentro da mesma linha de pesquisa com outras plantas medicinais. Mattos (2000a) estudou a produção de matéria seca e de óleo essencial por plantas de Mentha arvensis L. (hortelãjaponesa), com doses de $0 ; 2 ; 4 ; 6$ e 8 $\mathrm{kg} / \mathrm{m}^{2}$ de adubo orgânico. O autor obteve produções máximas, nas duas variáveis, com $6 \mathrm{~kg} / \mathrm{m}^{2}$ de adubação. Em experimento similar, Cruz (1999) também obteve, para Mentha x villosa Huds. (hortelã-rasteira), produções máximas com $6 \mathrm{~kg} / \mathrm{m}^{2}$ de adubação. Os dois pesquisadores mencionados trabalharam no Nordeste brasileiro.

Estudando os efeitos das doses de 0; 1; 2; 3 e 4 kg/m² de adubo orgânico em plantas de Achillea millefolium L. ("milfolhas”), Scheffer e Ronzelli Júnior
(1990), no Paraná, verificaram que as maiores produções de biomassa foram obtidas nos tratamentos que receberam 2 a $4 \mathrm{~kg} / \mathrm{m}^{2}$ de adubo e os maiores teores de óleo essencial com as doses de 1 a $4 \mathrm{~kg} / \mathrm{m}^{2}$. Ueda e Ming (1998), em Botucatu (SP), observaram correlação positiva da adubação química $(\mathrm{N}, \mathrm{P}, \mathrm{K})$ com a produção de biomassa, ao longo de um ano, por plantas de Cymbopogon winterianus (citronela-de-java), mas a produção de óleo essencial não foi afetada pela adubação.

As alturas de corte diferiram significativamente em relação à produção de matéria seca e de óleo essencial, nas duas colheitas (Tabela 2). Na primeira colheita, as produções de matéria seca obtidas nos cortes a 15 e $30 \mathrm{~cm}$ de altura foram de 0,944 e $0,839 \mathrm{t} / \mathrm{ha}$, respectivamente, e não diferiram significativamente entre si, mas foram superiores à obtida com corte a $45 \mathrm{~cm}$. Na segunda colheita ocorreu situação inversa, isto é, corte a $45 \mathrm{~cm}$ resultou na maior produção (1,840 t/ha), enquanto as outras produções obtidas foram inferiores e estatisticamente equivalentes. As produções totais de cada tratamento, representadas pelo somatório das produções obtidas nas duas colheitas, obedeceram ao mesmo padrão observado na segunda colheita: a maior produção $(2,562 \mathrm{t} / \mathrm{ha})$ foi obtida com corte a $45 \mathrm{~cm}$. Isto se deve à magnitude dos valores obtidos na segunda colheita em relação à primeira (provavelmente devido ao fato das 
Tabela 2. Efeito da altura de corte em plantas de Lippia alba, quimiotipo limoneno-carvona, em duas colheitas (agosto e outubro/2002). Pentecoste (CE), UFC, 2002.

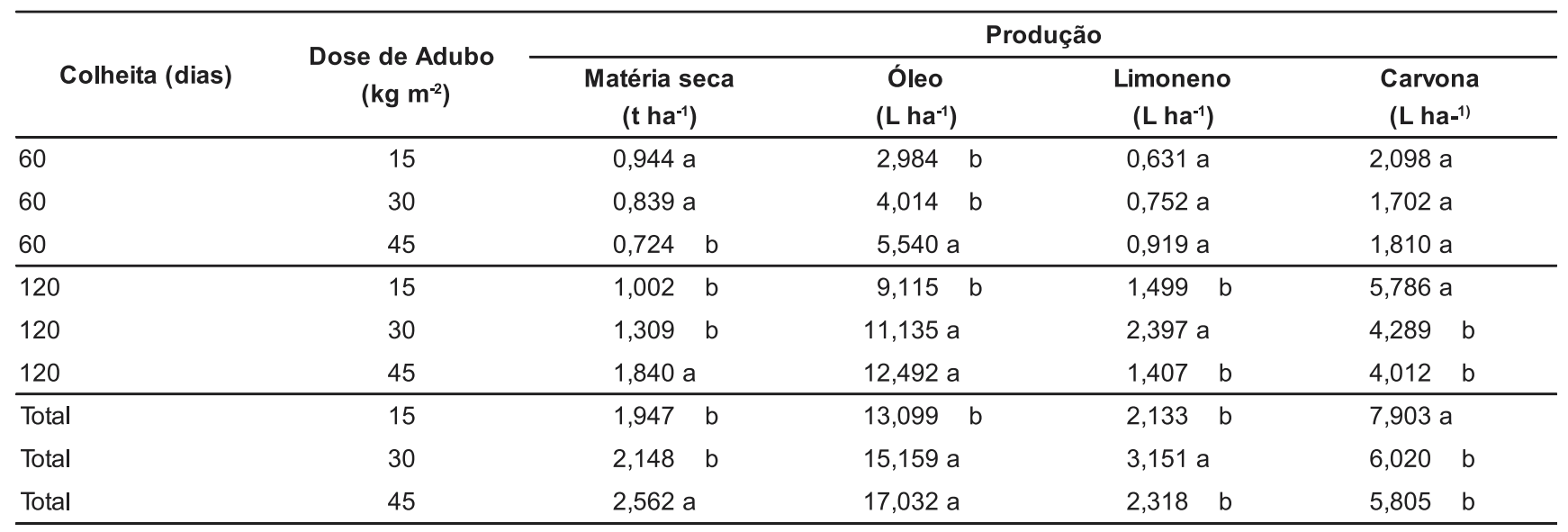

*/ Letras diferentes indicam diferença significativa, dentro de cada colheita, com 5\% de probabilidade pelo teste de Tukey.

plantas já estarem plenamente estabelecidas, como mencionado anteriormente). Em termos práticos, pode-se afirmar que as plantas cortadas a $45 \mathrm{~cm}$ de altura apresentaram crescimento muito mais intenso do que as outras plantas, a ponto de compensar os menores valores obtidos na primeira colheita.

Na segunda colheita, os rendimentos de óleo essencial presente nas folhas foram muito superiores aos obtidos na primeira colheita. O corte das plantas a $45 \mathrm{~cm}$ de altura resultou em maiores rendimentos de óleo essencial em relação aos outros tratamentos, na primeira colheita, obtendo-se uma produção de $5,540 \mathrm{~L} / \mathrm{ha}$. Na segunda colheita, os rendimentos obtidos nos cortes a 30 e 45 $\mathrm{cm}$ não diferiram significativamente entre si (11,135 e 12,492 L/ha, respectivamente) e foram superiores aos resultados obtidos no corte a $15 \mathrm{~cm}$. O mesmo padrão foi observado quando se considerou os valores obtidos nas duas colheitas conjuntamente, obtendo-se 15,159 e $17,032 \mathrm{~L} / \mathrm{ha}$, respectivamente, nos cortes a 30 e $45 \mathrm{~cm}$ de altura. Estes resultados refletem o fato confirmado por Castro (2001), de que nas porções apicais das plantas de Lippia alba a produção de metabólitos secundários é mais intensa do que em suas porções basais.

A altura de corte não influenciou a produção dos compostos estudados na primeira colheita. Porém, na segunda colheita e no total das duas colheitas, o corte à altura de $30 \mathrm{~cm}$ resultou nas produções máximas de limoneno $(2,397$ e 3,151 L/ha) e, na produção de carvona, os maiores resultados foram obtidos com o corte a $15 \mathrm{~cm}$ de altura $(5,805 \mathrm{e}$ $7,903 \mathrm{~L} / \mathrm{ha})$. A falta de uniformidade desses resultados reflete o dinamismo das interconversões que ocorrem continuamente entre os constituintes dos óleos essenciais. Estas interconversões envolvem reações de oxidação, redução, hidratação, desidratação, ciclização e isomerização, influenciadas por fatores ambientais (Castro, 2001). Resultado similar ocorreu no trabalho de Innecco et al. (2000), que submeteram plantas de Lippia sidoides (alecrim-pimenta) a quatro cortes sucessivos, a 10; 20; 30 e $40 \mathrm{~cm}$ de altura, observando que tanto as produções máximas de matéria seca quanto as de óleo essencial ocorreram em plantas cortadas a $30 \mathrm{~cm}$ de altura. Porém, Mattos et al. (2000), trabalhando com Ocimum gratissimum (alfavaca-cravo), realizaram cinco cortes, em intervalos de 30 dias, nas alturas de 15 e $25 \mathrm{~cm}$, observando que estes dois tratamentos não resultaram em diferenças significativas em relação às produções de matéria seca ou óleo essencial.

Nas condições do presente trabalho, com relação ao quimiotipo limonenocarvona da espécie Lippia alba (Mill.) N.E. Brown, pode-se afirmar que a adubação não influenciou significativamente a produção de biomassa foliar e de óleo essencial e não afetou a produção de limoneno, além de influenciar negativamente a produção de carvona. Entre as alturas de corte testadas, $45 \mathrm{~cm}$ é a que resultou na maior produção de biomassa foliar por área e, 30 e 45 cm são equivalentes quanto ao rendimento de óleo essencial por área. Porém, as maiores produções de limoneno podem ser obtidas com corte à altura de $30 \mathrm{~cm}$ e as de carvona com corte à altura de $15 \mathrm{~cm}$.

\section{LITERATURA CITADA}

ALENCAR, J.W.; CRAVEIRO, A.A.; MATOS, F.J.A. Kovats indexes as a preselection routine in mass-spectra library searches of volatiles. Journal of Natural Products, v.47, n.5, p.890-892, 1984. ANGELUCCI, M.E.M.; CORDAZZO, S.N.; FORTES, V.A. Efeitos farmacológicos do extrato de Lippia alba (Mill.) N.E.B. In: SIMPÓSIO DE PLANTAS MEDICINAIS DO BRASIL, 11., 1990, João Pessoa. Resumos... João Pessoa: SBPM, 1990. PN 4.12

CARLOS, I.C.C.; PESSOA, M.T.F.C.; SIQUEIRA, R.L.C.L. Registro de medicamentos fitoterápicos. Fortaleza: Secr. Saúde do Est. do Ceará, 2000. 37 p. CASTRO, D.M. Efeito da variação sazonal, colheita selecionada e temperaturas de secagem sobre a produção de biomassa, rendimento e composição de óleos essenciais de folhas de Lippia alba (Mill.) N. E. Br. ex Britt. e Wilson (Verbenaceae). 2001. 132 p. (Tese doutorado), UNESP, Botucatu.

CRAVEIRO, A.A.; FERNANDES, A.G.; ANDRADE, C.H.; MATOS, F.J.A.; ALENCAR, J.W.; MACHADO, M.I.L. Óleos essenciais de plantas do nordeste. Fortaleza: EUFC, 1981. 209 p.

CRUZ, G.F. Desenvolvimento de sistema de cultivo para hortelã-rasteira (Menthax villosa Huds.). 1999. 35 p. (Tese mestrado), UFC, Fortaleza.

GOMES, F.P. Curso de estatística experimental. São Paulo: Nobel, 1986. 430 p.

INNECCO, R.; MATTOS, S.H.; CRUZ, G.F. Determinação da altura de corte do alecrim-pimenta. Horticultura Brasileira, Brasília, v.18, p.992993, 2000. Suplemento. Trabalho apresentado no $40^{\circ}$ Congresso Brasileiro de Olericultura, 2000. 
MATOS, F.J.A. As ervas cidreiras do nordeste do Brasil: estudo de três quimiotipos de Lippia alba (Mill.) N.E. Brown (Verbenaceae). Parte II Farmacoquímica. Revista Brasileira de Farmácia, v.77, n.4, p.137-141, 1996.

MATOS, F.J.A. O formulário fitoterápico do professor Dias da Rocha. 2. ed. Fortaleza: EUFC, 1997. p.124.

MATTOS, S.H. Estudos fitotécnicos da Mentha arvensis L. var. Piperacens Holmes como produtora de mentol no Ceará. 2000a. 98 p. (Tese doutorado), UFC, Fortaleza.

MATTOS, S.H. Perspectivas do cultivo de plantas medicinais para a fitoterapia no Estado do Ceará Horticultura Brasileira, Brasília, v.18, p.45-46, 2000. Suplemento. Trabalho apresentado no $40^{\circ}$ Congresso Brasileiro de Olericultura, 2000b.

MATTOS, S.H.; INNECCO, R.; CRUZ, G.F.; EHLERT, P.A.D. Determinação da altura de corte em alfavaca-cravo. Horticultura Brasileira, Brasília, v.18, p.992-993, 2000. Suplemento. Trabalho apresentado no $40^{\circ}$ Congresso Brasileiro de Olericultura, 2000.
MING, L.C. Estaquia da falsa erva cidreira - Lippia alba N. E. Brown (Verbenaceae). In: SIMPÓSIO DE PLANTAS MEDICINAIS DO BRASIL, 11. 1990, João Pessoa. Resumos... João Pessoa: SBPM, 1990. PN 4.80.

MING, L.C. Influência de diferentes niveis de adubação orgânica na produção de biomassa e teor de óleos essenciais de Lippia alba (Mill.) N.E. Br. Verbenaceae. 1992. 206 p. (Tese mestrado), UFPR, Curitiba.

RETAMAR, J.A. Characteristics of essential oils. Rivista Italiana Essenze, Profumi, Piante Officinali, Aromi, Saponi, Cosmetici, Aerosol, v.59, n.10, p.534-537, 1977. Disponível em: $<$ http://www.periodicos.capes.gov.br $>$. Acesso em 29/10/2002.

SCHEFFER, M.C.; RONZELLI JÚNIOR, P. Influência de diferentes níveis de adubação orgânica sobre a biomassa e teor de óleos essenciais da Achillea millefolium L. In: SIMPÓSIO DE PLANTAS MEDICINAIS DO BRASIL, 11., 1990, João Pessoa. Resumos... João Pessoa: SBPM, 1990. PN 4.12 .
UEDA, E.T.; MING, L.C. Influência de N, P, K na produção de biomassa foliar e teor de óleo essencial em citronela-de-java - Cymbopogon winterianus - Poaceae. In: CONGRESSO BRASILEIRO DE OLERICULTURA, 38., 1998, Petrolina. Resumos... Petrolina: SOB, 1998. p. 352. ZOGHBI, M.G.B.; ANDRADE, E.H.A.; SANTOS, A.S.; SILVA, N.H.L.; MAIA, J.G.S Essential oils of Lippia alba (Mill.) N.E.Br. growing wild in the brazilian amazon. Flavour and Fragrance Journal, v.13, n.1, p.47-48, 1998. Disponível em: <http:// www.periodicos.capes.gov.br>. Acesso em: 29/10/ 2002. 